\title{
Synergistic anticancer and antibacterial activities of cordycepin and selected natural bioactive compounds
}

\author{
$\mathrm{He} \mathrm{Ni}^{1}$, Rui-Lin Hao ${ }^{1}$, Xue-Feng $\mathrm{Li}^{1}$, Vassilios Raikos ${ }^{2}$, Hai-Hang $\mathrm{Li}^{1,3}$ \\ ${ }^{1}$ Guangdong Provincial Key Lab of Biotechnology for Plant Development, School of Life Sciences, South China Normal \\ University, Guangzhou 510631, ${ }^{2}$ Rowett Institute, University of Aberdeen, Aberdeen, AB25 2ZD, Scotland, UK, ${ }^{3}$ Institute of \\ Brain Science and Rehabilitation Medicine, South China Normal University, Guangzhou 510631, China
}

*For correspondence: Email: xa1209@163.com; Tel: +862085212630

Sent for review: 29 May 2018

Revised accepted: 29 July 2018

\begin{abstract}
Purpose: To investigate if cordycepin (Cor) exerts synergistic anticancer and antibacterial activities with curcumin (Cur), resveratrol (Res), genistein (Gen), and anthocyanidin (Ant) in order to improve its therapeutic effect.

Methods: Antibacterial activity was assayed against Escherichia coli BL21 (DE3) cultured in the LuriaBertani (LB) medium, while anticancer activity against Hela cells was determined using 3-(4, 5dimethylthiazol-2-yl)-2, 5-diphenyltetrazolium bromide (MTT) assay. Immunofluorescence assay was used to measure the expressions of p53 and caspase-3, while apoptosis was assayed in Hela cells using Wright-Giemsa dye staining method.

Results: The combination of Cor and Gen produced the most significant synergistic antibacterial activity, while combination of Cor, Cur and Gen exerted significant and synergistic anticancer activity against Hela cells. Among all three-compound combinations, Cor + Res + Gen had the highest synergistic anticancer activity. Immunofluorescence data indicate that Cor functioned by up-regulating p53-dependent apoptosis, while Res exerted its effect by p53- and caspase-3-dependent apoptosis, and Gen functioned by inhibiting adenosine deaminase and Cor degradation.

Conclusion: The three compounds function via different mechanisms, and synergistically induce Hela cell apoptosis. This work provides a strong basis for the development of highly active and multicomponent anticancer drugs from natural sources.
\end{abstract}

Keywords: Cordycepin, Natural phenolic compounds, Caspase, Anticancer, Synergy

\begin{abstract}
This is an Open Access article that uses a funding model which does not charge readers or their institutions for access and distributed under the terms of the Creative Commons Attribution License (http://creativecommons.org/licenses/by/4.0) and the Budapest Open Access Initiative (http://www.budapestopenaccessinitiative.org/read), which permit unrestricted use, distribution, and reproduction in any medium, provided the original work is properly credited.

Tropical Journal of Pharmaceutical Research is indexed by Science Citation Index (SciSearch), Scopus, International Pharmaceutical Abstract, Chemical Abstracts, Embase, Index Copernicus, EBSCO, African Index Medicus, JournalSeek, Journal Citation Reports/Science Edition, Directory of Open Access Journals (DOAJ), African Journal Online, Bioline International, Open-J-Gate and Pharmacy Abstracts
\end{abstract}

\section{INTRODUCTION}

Natural bioactive compounds have been shown to exhibit various therapeutic effects, such as anticancer [1,2], antibacterial [3], antioxidant [4] and anti-diabetic properties [5]. Bioactive compounds derived from natural sources show diversity in terms of chemical structures, biological activities and mechanisms of action. However, most natural compounds have disadvantages such as relatively low efficacy, delayed activity at the site of action and/or fast degradation which restricts their potential applications as clinical drugs [6]. 
Numerous reports indicate that bioactive compounds with varying chemical structures may act at different drug targets through multiple mechanisms or signaling pathways for the same disease. They may synergistically function as multi-target drugs at low doses [7]. Synergistic interactions are common between anticancer polyphenols, and also between anticancer chemotherapeutic drugs and phenolic compounds [8]. It has been demonstrated that the combination of Cur $(20 \mu \mathrm{mol} / \mathrm{L})$ and Gen (100 $\mu \mathrm{mol} / \mathrm{L})$ effectively inhibited angiogenesis in human prostate cancer cells, as indicated by significant decline in the expressions of ARNT and HIF-1a protein [9].

Apigenin and Cur produce synergistic anticancer activity by binding at different sites of tubulin [10]. The combination of Cur and ellagic acid at various concentrations elicited better anticancer properties than when used singly [11]. It has been reported that cucurbitacin-induced apoptosis in human hepatoma cells was synergistically enhanced by Cur, which also reversed cucurbitacin-induced multidrug resistance in these cells [12]. Combination of Res and thymoquinone synergistically reduced tumor growth in breast cancer mice by induction of apoptosis, inhibition of angiogenesis, and immune modulation [13].

Cordycepin (Cor) is an important bioactive compound isolated from Cordyceps militaris. It has anticancer, antibacterial, and immuneregulatory properties [14]. However, many studies have shown that the effectiveness of Cor in vivo is limited due to its rapid deamination by adenosine deaminase [15,16]. Preliminary studies have identified a number of phenolic compounds which inhibit the activity of adenosine deaminase [17]. In the present study, the antibacterial and anticancer activities of Cor in combination with several bioactive phenolic compounds, were investigated.

\section{EXPERIMENTAL}

\section{Preparation of natural products}

Cordycepin (Cor) was prepared as described previously [14]. Curcumin (Cur), Res, Gen, and Ant were purchased from Qiyun Biotechnology Ltd, China. Antibodies for p53 and caspase-3, and Goat anti-Rabbit IgG/FITC were purchased from Boster Biological Technology Co. Ltd, China. These compounds were more than $98 \%$ pure, and were dissolved in water or $4 \%(\mathrm{v} / \mathrm{v})$ ethanol prior to use. This research was approved by the Ethical Committee of Guangdong Provincial Key Lab of Biotechnology for Plant Development, School of Life Sciences, South
China Normal University, Guangzhou 510631, China according to the declaration of Helsinki promulgated in 1964 as amended in 1996, the approval number is 2804 [18].

\section{Antibacterial assay}

Escherichia coli BL21 (DE3) was cultured in the Luria-Bertani (LB) medium for $3 \mathrm{~h}$ at $37^{\circ} \mathrm{C}$. Then, different concentrations of Cor, Cur, Res, Gen, and Ant were added to the inoculum and cultured for another $6 \mathrm{~h}$. The absorbance of the culture medium suspension (as a measure of bacterial growth) was read at $600 \mathrm{~nm}$ in a spectrophotometer (model 6105, Jenway, Essex, UK).

\section{Evaluation of anticancer activity}

The anticancer activities of Cor, Cur, Res, Gen, and Ant were determined using standard 3-(4, 5dimethylthiazol-2-yl)-2, 5- diphenyltetrazolium bromide (MTT) assay. Hela cells were grown in 96-well plates at the concentration of 20,000 cells/well in $200 \mu \mathrm{L}$ (Roswell Park Memorial Institute (RPMI 1640) medium. The cells were allowed to attach overnight, after which various concentrations of Cor, Cur, Res, Gen, and Ant were added to separate wells. At 24,48 , and 72 h of incubation, $20 \mu \mathrm{L}$ of $0.5 \mathrm{mg} / \mathrm{ml}$ MTT reagent (Sigma-Aldrich Co. Ltd., Poole, UK) was added to each well. After $4 \mathrm{~h}$ incubation, the culture solutions were removed, and $500 \mu \mathrm{L}$ of dimethyl sulfoxide (DMSO) solution was added to each well to solubilize the formazan crystals formed. The absorbance of each well was read at $530 \mathrm{~nm}$ in an Ultra Multifunctional Microplate Reader 680 (Bio-Rad, Hercules, CA, USA). The experiment was repeated three times.

\section{Determination of apoptosis}

Apoptosis was assayed in Hela cells stained with Wright-Giemsa dye solution. After treatment with Cor, Cur, Res, Gen, and Ant for $48 \mathrm{~h}$, the cells were stained with Wright-Giemsa dye solution according to the manufacturer's instructions, and examined using light microscopy $[19,20]$.

\section{Determination of p53 and caspase-3 expressions by immunofluorescence}

Following treatment with Cor, Cur, Res, Gen, and Ant, Hela cells were rinsed twice in phosphate buffered saline (PBS), fixed in $100 \mu \mathrm{L} 4 \%$ paraformaldehyde for 30 min, rehydrated in PBS, and incubated with the primary antibodies overnight at $4{ }^{\circ} \mathrm{C}$. Thereafter, the cells were rinsed with PBS and incubated with anti-rabbit IgG, followed by streptavidin-conjugated FITC (1: 
200 dilution in blocking solution) for $2 \mathrm{~h}[21,22]$. After washing with PBS, p53 and caspase-3 expressions were assessed under inverted fluorescence microscopy (Eclipse TE2000-U, Nikon Tokyo, Japan).

\section{Statistical analysis}

The results are expressed as mean \pm standard deviation (SD) of at least three replicates. Statistical analysis of the data was performed using SPSS software, version 22 (IBM). Data were analyzed by analysis of variance (ANOVA) and significant differences $(p<0.05)$ were detected by Scheffé's post hoc test.

\section{RESULTS}

\section{Antibacterial activity of individual compounds}

As shown in Figure 1, Gen had the highest antibacterial activity; it inhibited microbial growth by $60 \%$ at $200 \mu \mathrm{mol} / \mathrm{L}$. A similar inhibition efficacy was produced by Cur $(57 \%)$ at 100 $\mu \mathrm{mol} / \mathrm{L}$, but its activity did not increase further when the concentration increased to $200 \mu \mathrm{mol} / \mathrm{L}$. Moderate and concentration-dependent inhibitions were exhibited by Cor and Ant against the growth of $E$. coli. The only compound that did not produce dose-dependent antibacterial effect was Res. It inhibited bacterial growth by $37 \%$ at a dose of $50 \mu \mathrm{mol} / \mathrm{L}$, but its inhibitory effect decreased at higher concentrations (100 and 200 $\mu \mathrm{mol} / \mathrm{L}$ ). The antibacterial activities of the five compounds tested followed the order Gen $>$ Cur $>$ Cor $>$ Ant $>$ Res.

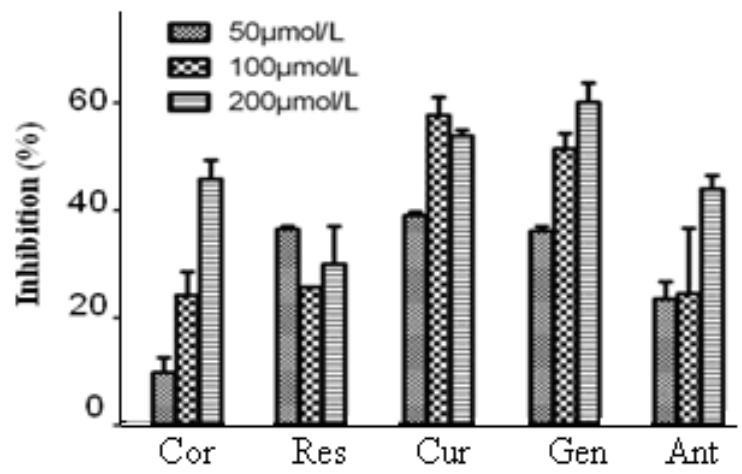

Figure 1: Inhibition of $E$. coli growth by Cor and phenolic compounds at different concentrations

\section{Cor produced synergistic antibacterial activity with phenolic compounds}

The results shown in Figure 2 demonstrate that Cor produced relatively low antibacterial activity. In order to improve its activity, it was used in combination with other tested compounds to see if the combinations would produce synergistic antibacterial activities. The combination of Cor and Gen at $50 \mu \mathrm{mol} / \mathrm{L}$ produced significant synergistic antibacterial activity, reflected in $60 \%$ inhibition of microbial growth $(p<0.05)$. This was 450 and $77 \%$ higher than the activity shown individually by Cor and Gen, respectively. Other compounds such as Res, Cur and Ant did not show any synergistic antibacterial activity and the activities of their combinations with Cor were even lower than values obtained when they were tested individually.

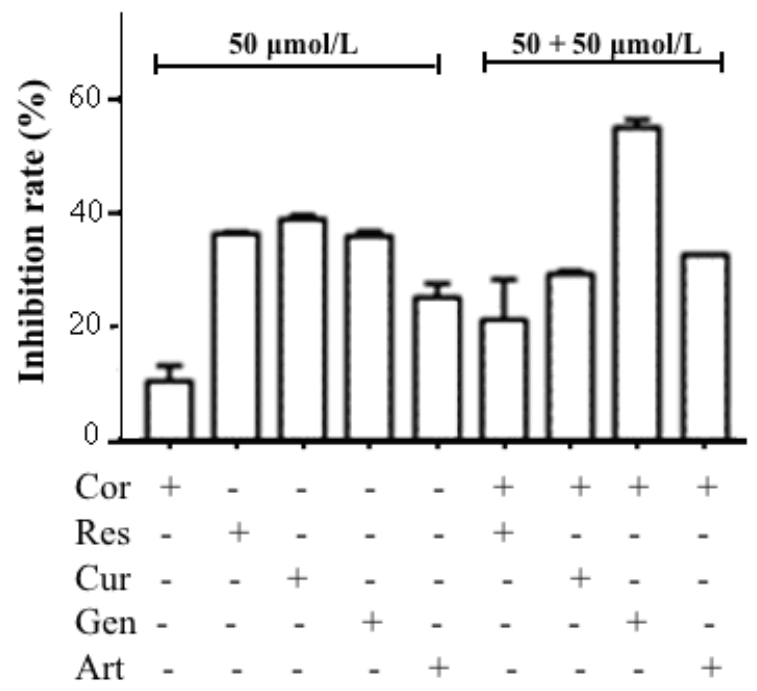

Figure 2: Inhibition of $E$. coli growth by Cor in combination with phenolic compounds at $50 \mu \mathrm{mol} / \mathrm{L}$

\section{Anticancer activity of Cor and phenolic compounds}

The anticancer activities of individual compounds (Cor, Res, Cur, Gen, and Ant) against Hela cells were determined at concentrations of $5,25,50$, 100 , and $200 \mu \mathrm{mol} / \mathrm{L}$. As shown in Figure 3, all the compounds significantly inhibited Hela cell in a dose- and time-dependent manner. The highest anticancer activity was produced by Cur, with approximately $90 \%$ growth inhibition in 72 $\mathrm{h}$, at concentrations higher than $100 \mu \mathrm{mol} / \mathrm{L}$. The inhibitions produced by the other compounds followed the order Res $>$ Ant $>$ Cor $>$ Gen.

\section{Cor showed synergistic anticancer activity with phenolic compounds}

Significant synergistic inhibition of the growth of Hela cells was produced by a combination of Cor, Cur and Gen at $50 \mu \mathrm{mol} / \mathrm{L}$, when compared with the inhibitions produced by single compounds (Figure 4). The inhibition by combination of Cor and Cur increased more than $75 \%$ and $17 \%$, relative to single Cor and Cur treatments, respectively. The inhibitory effect by Cor + Gen mixture increased by more than $50 \%$, 
when compared to individual Cor or Cur treatments at the same dose $(50 \mu \mathrm{mol} / \mathrm{L})$. However, the combination of Cor with Res or Ant failed to produce any synergistic inhibitory effects on the growth of Hela cells.

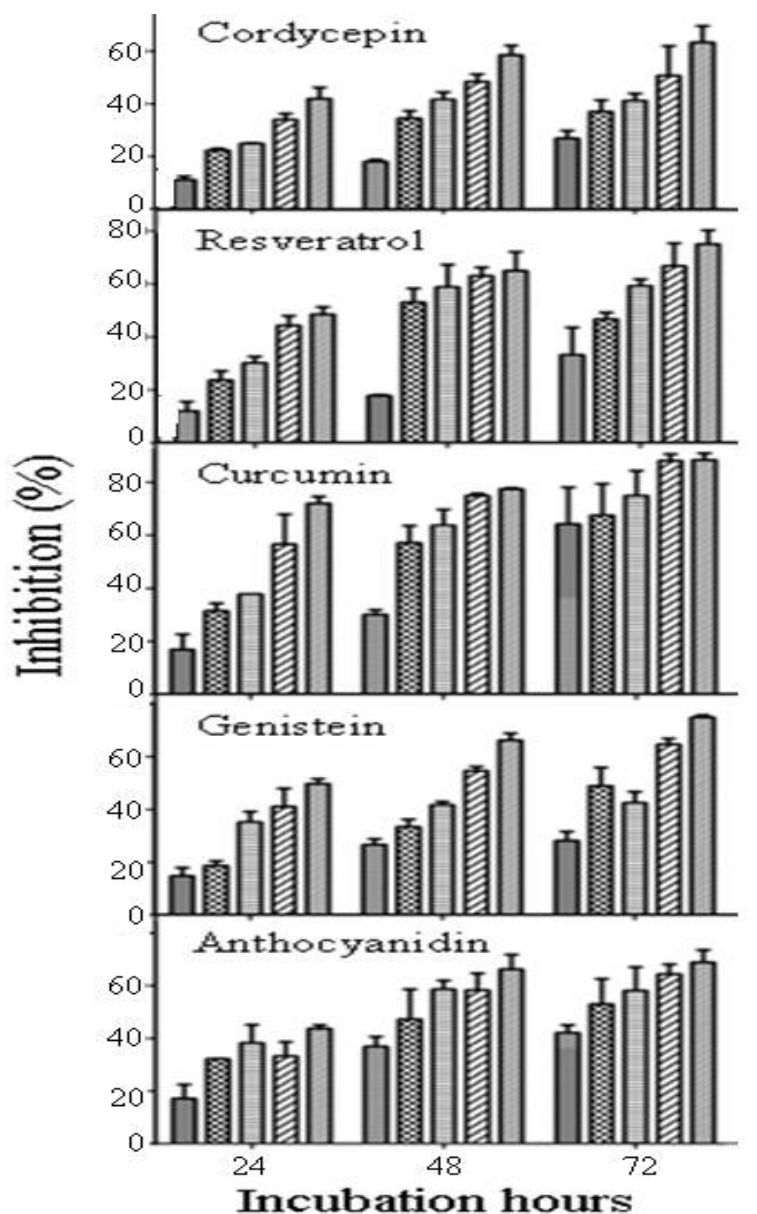

Figure 3: Inhibition on Hela cells at $5(\mathbf{Q}), 25(\mathbf{\vec { N }})$, 50(=), $100($. .), and $200(\% / /) \mu \mathrm{mol} / \mathrm{L}$ for 24,48 , and 72 $\mathrm{h}$ by Cor and phenolic compounds

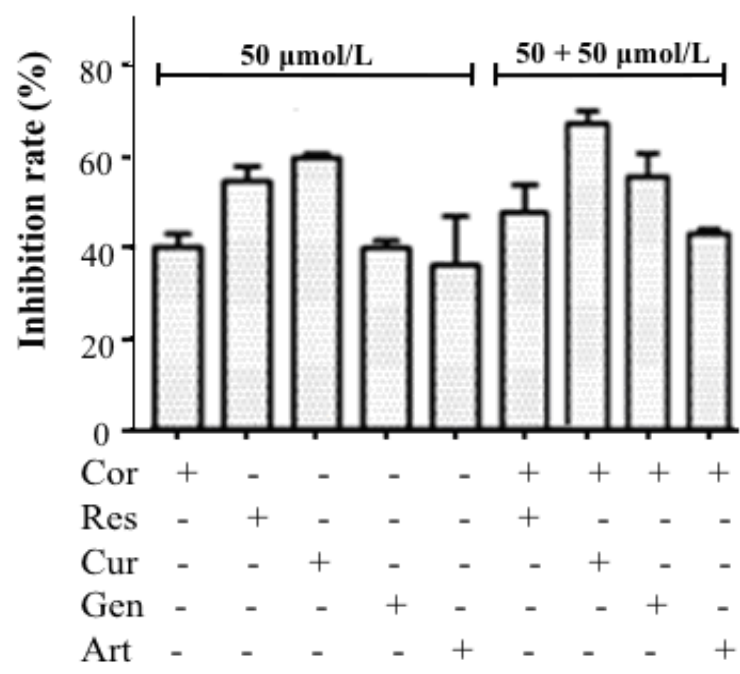

The synergistic anticancer activities of Cor with two phenolic compounds were also investigated at a reduced dosage of $25 \mu \mathrm{mol} / \mathrm{L}$. The results in Figure $5 \mathrm{~A}$ showed that all five three-compound combinations yielded synergistic anticancer activities, which were significantly higher than those produced by their components when used singly. The three-component combination of Cor, Res and Gen $(25 \mu \mathrm{mol} / \mathrm{L})$ had the highest anticancer activity, which was considerably higher than that produced by Cor, Res or Gen when tested alone. A similar pattern of results was obtained with respect to the effects of the treatments on apoptosis (Figure 5B). When compared to the control and treatments with individual compounds, Hela cell volume and number were significantly decreased in the combined treatment with Cor, Res and Gen. Hela cells showed nuclear condensation and early apoptosis after 48-h treatment, which indicated that the combined treatment with Cor, Res and Gen exerted significant synergistic anticancer activities $(p<0.05)$.

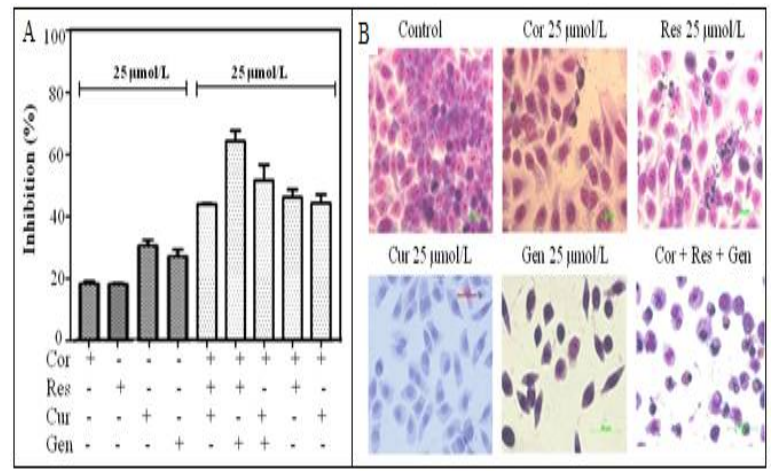

Figure 5: Synergistic inhibition of Hela cells by Cor in combination with two phenolic compounds at 25 $\mu \mathrm{mol} / \mathrm{L}$

\section{Caspase- 3 and p53 expressions}

The expression of p53 and Caspase-3 were studied in the combined treatment of three compounds (Cor + Res + Gen) with immunofluorescence analysis to investigate the mechanism involved in their anticancer activities. As shown in Figure 6, Cor and Res up-regulated p53 expressions, whereas Gen did not produce a similar effect. The combination of Cor, Res and Gen synergistically produced higher level of upregulation in p53 expression. As shown in Figure 7, Cor and Gen did not induce caspase-3 expression, while Res up-regulated it. However, the combination of Cor, Res and Gen exerted a synergistic effect by up-regulating caspase-3 expression and inducing Hela cell apoptosis.

Figure 4: Synergistic inhibition on the growth of Hela cells at $50 \mu \mathrm{mol} / \mathrm{L}$ by Cor in combination with phenolic compounds 

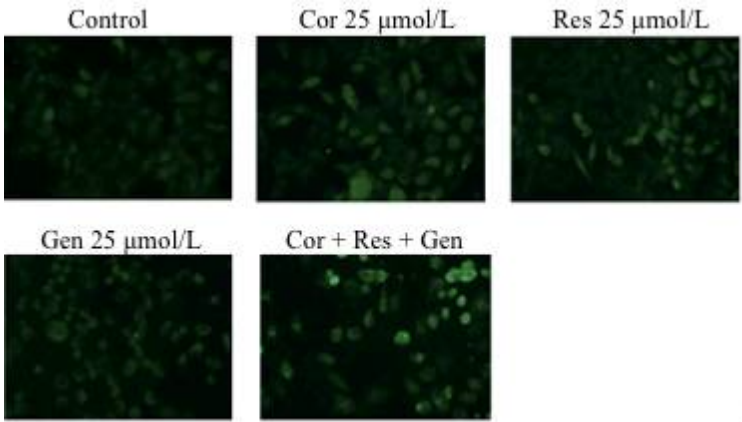

Figure 6: Immunofluorescence analysis of p53 expression after treatment for $48 \mathrm{~h}$

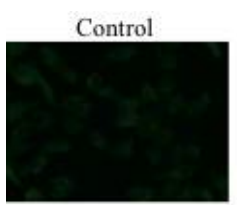

Gen $25 \mu \mathrm{mol} / \mathrm{L}$

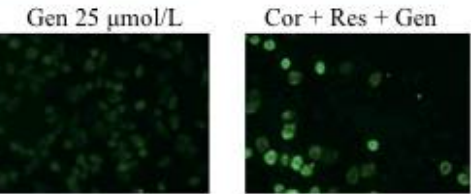

Figure 7: Immunofluorescence analysis of caspase-3 expression after treatment with different natural products for $48 \mathrm{~h}$

\section{DISCUSSION}

Although Res inhibited bacterial growth by $37 \%$ at a dose of $50 \mu \mathrm{mol} / \mathrm{L}$, its inhibitory effect decreased at higher concentrations. Indeed, Res was the only compound that failed to produce dose-dependent antibacterial activity. Low inhibitory activity of Res against gram-positive bacteria has been previously reported [23].

The only significant synergistic antibacterial effect was produced by combination of Cor and Gen at $50 \mu \mathrm{mol} / \mathrm{L}$, which resulted in much higher antibacterial effect than when the compounds were used singly. It has been previously reported that Cor is an $\mathrm{NAD}^{+}$-dependent DNA ligase inhibitor which inhibits bacterial growth [24], whereas Gen inhibits DNA and protein synthesis in Klebsiella pneumonia [25]. Therefore, Cor and Gen may mediate their antibacterial activities through different mechanisms, which manifested in a synergistic inhibitory effect on E.coli growth. Furthermore, Gen is known to inhibit the activity of adenosine deaminase [17]. Thus, it may function by inhibiting the degradation of Cor in cells.

The results of combination of Cor, Res and Gen suggest that Cor and Res can induce p53 expression and activate the p53-dependent apoptotic pathway. Res could also up-regulate caspase- 3 and activate the caspase- 3 dependent apoptotic pathway. On the other hand, Gen did not show any activity on both p53- and caspase3-dependent pathways. Since Gen is able to inhibit adenosine deaminase activity, it may function by inhibiting the degradation of Cor [17,31]. All three natural compounds may have acted on both p53- and caspase-3-dependent apoptotic pathways, thereby inhibiting the growth of Hela cells in a synergistic fashion.

A large body of evidence indicates that Cor exerts significant in vitro anticancer activity against different cancer cell lines [26,27]. However, the therapeutic effect of Cor on human cancer cells is not ideal due to its fast in vivo degradation by adenosine deaminase. In order to improve its inhibitory effect and reduce dosage, synergistic anticancer activities of Cor with the phenolic compounds were investigated.

As a regulator of the apoptotic process, p53 modulates key control points in both the extrinsic and intrinsic pathways. Up-regulation of p53 expression in tumor cells can induce apoptosis $[28,29]$. In mammals, caspases appear to be activated in a protease cascade that leads to inappropriate activation or rapid disablement of key structural proteins such as important enzymes involved in signaling, homeostasis and repair. Caspase- 3 is a frequently activated death protease. It is a key protease in apoptosis, and an important target in cancer therapy [30].

\section{CONCLUSION}

The results obtained in this study show that the combination of Cor and Gen exerts synergistic antibacterial activities, while the combination of Cor, Res and Gen produces significant synergistic anticancer activities through different apoptosis-related pathways. These findings demonstrate that different natural bioactive compounds can be combined to act as a multicomponent drugs which synergistically inhibit bacterial and cancer cell growth at relatively low concentrations. These results provide useful clues for the development of new drugs with potential anticancer activities from natural compounds.

\section{DECLARATIONS}

\section{Acknowledgement}

This study was supported by the Guangdong Provincial Science and Technology Programs (no. 2014A010107026), China Central Government Agricultural Technology Promotion no. 2015-60-43, Natural Science Funds of Guangdong Province (Program no. 
2017A030310031) and Guangdong Special Funds for Development of Science and Technology Innovation of University Students (Program no. pdjh2015b0146).

\section{Conflict of interest}

No conflict of interest associated with this work

\section{Contribution of authors}

We declare that this work was done by the author(s) named in this article and all liabilities pertaining to claims relating to the content of this article will be borne by the authors, all authors read and approved the manuscript for publication. Hai-Hang Li conceived and designed the study, $\mathrm{He} \mathrm{Ni}$, Rui-Lin Hao, Xue-Feng Li, Vassilios Raikos, Hai-Hang Li collected and analysed the data, He Ni wrote the manuscript.

\section{REFERENCES}

1. Crotti $S$, Posocco B, Marangon E, Nitti D, Toffoli G, Agostini M. Mass spectrometry in the pharmacokinetic studies of anticancer natural products. Mass Spectrom Rev 2017; 36(2): 213-251.

2. Curti V, Lorenzo AD, Dacrema M, Xiao J, Nabavi SM, Daglia M. In vitro polyphenol effects on apoptosis: An update of literature data. Semin Cancer Biol 2017; 46 : 119-131.

3. Mbaveng AT, Sandjo LP, Tankeo SB, Ndifor $A R$, Pantaleon A, Nagdjui BT, Kuete V. Antibacterial activity of nineteen selected natural products against multi-drug resistant Gram-negative phenotypes. Springer Plus 2015; 4: 823.

4. Geoffroy TR, Meda NR, Stevanovic T. Suitability of $D P P H$ spiking for antioxidant screening in natural products: the example of galloyl derivatives from red maple bark extract. Anal Bioanal Chem 2017; 409(22): 5225-5237.

5. Hung HY, Qian K, Morrisnatschke SL, Hsu CS, Lee KH. Recent discovery of plant-derived anti-diabetic natural products. Nat Prod Rep 2012; 29 (5): 580.

6. Harvey AL. Natural products in drug discovery. Drug Discov Today 2008; 13(19): 894-901.

7. Rejhova A, Opattova A, Cumov A, Sliva $D$, Vodička P. Natural compounds and combination therapy in colorectal cancer treatment. Eur J Med Chem 2018; 144: 582-594.

8. Lewandowska U, Gorlach S, Owczarek K, Hrabec E, Szewczyk K. Synergistic interactions between anticancer chemotherapeutics and phenolic compounds and anticancer synergy between polyphenols. Postepy Hig Med Dosw (Online) 2014; 68: 528-540.

9. Aditya NP, Shim $M$, Yang $H$, Lee YJ, Ko $S$. Antiangiogenic effect of combined treatment with curcumin and genistein on human prostate cancer cell line. J Funct Food 2014; 8: 204-213.

10. Choudhury D, Ganguli A, Dastidar DG, Acharya BR, Das A, Chakrabarti G. Apigenin shows synergistic anticancer activity with curcumin by binding at different sites of tubulin. Biochimie 2013; 95: 1297-1309.

11. Kumara D, Basua $S$, Parijab L, Rout D, Manna $S$, Dandapat J, Debata PR. Curcumin and Ellagic acid synergistically induce ROS generation, DNA damage, p53 accumulation and apoptosis in HeLa cervical carcinoma cells. Biomed Pharmacother 2016; 81: 3137.

12. Sun $Y$, Zhang J, Zhou J, Huang Z, Hu H, Qiao M, Zhao $X$, Chen $D$. Synergistic effect of cucurbitacin $B$ in combination with curcumin via enhancing apoptosis induction and reversing multidrug resistance in human hepatoma cells. Eur J Pharmacol 2015; 768: 28-40.

13. Omar HA, Wamidh HT, Iman AB. Antitumor effect of thymoquinone combined with resveratrol on mice transplanted with breast cancer. Asian Pac J Trop Med 2017; 10(4): 400-408.

14. $\mathrm{Ni} \mathrm{H}$, Zhou XH, Li HH, Huang WF. Column chromatographic extraction and preparation of cordycepin from Cordyceps militaris waster medium. $J$ Chromatogr B Analyt Technol Biomed Life Sci 2009; 877(22): 2135-2141.

15. Chang HM, Oakes J, Olsson A, Panaitescu L, Britt BM, Kearney CM, Kane RR. Synthesis and in vitro evaluation of adenosine deaminase resistant $N-6$ aminal and thioaminal prodrugs of cordycepin. Lett Drug Des Discov 2005; 2(2): 133-136.

16. Li G, Izumi N, Shuichi H, Itoh T, Fujiwara R. Inhibition of adenosine deaminase (ADA)-mediated metabolism of cordycepin by natural substances. Pharmacol Res Perspect 2015; 3(2): 00121.

17. Ni H, Li YH, Hao RL, Li H, Hu SQ, Li HH. Identification of adenosine deaminase inhibitors from Tofu wastewater and litchi peel and their synergistic anticancer and antibacterial activities with cordycepin. Int $\mathrm{J}$ Food Sci Technol 2016; 51(5): 1168-1176.

18. World Health Organization. Declaration of Helsinki. Br Med J 1996; 313(7070): 1448-1449.

19. Ling YH, Tornos C, Perez-Soler R. Phosphorylation of $\mathrm{BCl}-2$ is a marker of $M$ phase events and not a determinant of apoptosis. J Biol Chem 1998; 273(30): 18984-18991.

20. Yu C, Rahmani M, Conrad D, Subler M, Dent P, Grant S. The proteasome inhibitor bortezomib interacts synergistically with histone deacetylase inhibitors to induce apoptosis in Bcr/Abl+ cells sensitive and resistant to STI571. Blood 2003; 102(10): 3765-3774.

21. Midgley $C$, Fisher $C$, Bartek J, Vojtĕsek B, Lane $D$, Barnes DM. Analysis of p53 expression in human tumours: an antibody raised against human p53 expressed in Escherichia coli. J Cell Sci 1992; 101(1): 183-189.

22. Sansome C, Zaika A, Marchenko ND, Moll UM. Hypoxia death stimulus induces translocation of $p 53$ protein to

Trop J Pharm Res, August 2018; 17(8): 1626 
mitochondria: Detection by immunofluorescence on whole cells. FEBS Lett 2001; 488(3): 110-115.

23. Tegos G, Stermitz FR, Lomovskaya O, Lewis $K$. Multidrug pump inhibitors uncover remarkable activity of plant antimicrobials. Antimicrob Agents Chemothrapy 2002; 10: 3133-3141.

24. Zhou XF, Cai GQ, He Y, Tong G. Separation of cordycepin from Cordyceps militaris fermentation supernatant using preparative HPLC and evaluation of its antibacterial activity as an NAD+-dependent DNA ligase inhibitor. Exp Ther Med 2016; 12: 1812-1816.

25. Duraiarasan S, Jayachandran K, Sekar N, Karthiga J. Isolation of genistein from Acalypha fruticosa and studying its antibacterial activity by inhibition of bacterial DNA and protein. J Herb Med Toxicol 2011; 5 (1): 8796.

26. Chaicharoenaudomrung $N$, Jaroonwitchawan $T$, Noisa $P$. Cordycepin induces apoptotic cell death of human brain cancer through the modulation of autophagy. Toxicol In vitro 2018; 46: 113-121.

27. Nakamura K, Shinozuka K, Yoshikawa N. Anticancer and antimetastatic effects of cordycepin, an active component of Cordyceps sinensis. J Pharmacol Sci 2015; 127: 53-56.

28. Feng Z, Hu W, Rajagopal G, Levine AJ. The tumor suppressor p53: cancer and aging. Cell Cycle 2008; 7(7): 842-847.

29. Fridman JS, Lowe SW. Control of apoptosis by $p 53$. Oncogene 2003; 22(56): 9030-9040.

30. Porter AG, Jänicke RU. Emerging roles of caspase-3 in apoptosis. Cell Death Differ 1999; 6(2): 99.

31. Jaques JAdS, Ruchel JB, Schlemmer KB, Pimentel VC, Bagatini M, Souza Vdo C, Moretto MB, Morsch VM, Schetinger MR, Leal DB. Effects of curcumin on the activities of the enzymes that hydrolyse adenine nucleotides in platelets from cigarette smoke-exposed rats. Cell Biochem Funct 2011; 29(8): 630-635. 